\title{
Snow Microwave Reflection at Mobile Phone Frequency Bands
}

\author{
Tarmo Koppel $^{1}$, Inese Vilcane ${ }^{2}$, Piia Tint ${ }^{1}$, Andrei Shiskin ${ }^{3}$ \\ ${ }^{1}$ Department of Work Environment and Safety, Tallinn University of Technology, \\ Ehitajate tee 5, 19086 Tallinn, Estonia, \\ ${ }^{2}$ Riga Technical University, Institute of Occupational Safety and Civil Defense, \\ Kalnciema street 6, Riga, LV-1048, Latvia, \\ ${ }^{3}$ Riga Technical University, Rudolfs Cimdins Riga Biomaterials Innovations and Development Centre Pulka \\ street 3, Riga, LV-1007, Latvia \\ Send correspondence to tarmo.koppel@ttu.ee
}

\begin{abstract}
Snow should be accounted in electromagnetic fields' risk assessment, especially in Nordic countries where in winter months snow precipitation and coverage may significantly affect the propagation of microwaves. The risk assessment should be always carried out under worst case scenario conditions, including the reflective properties of the surfaces surrounding the antenna. In this study fresh snow microwave reflectance properties were investigated at the mobile communication bands from 1700 to $2700 \mathrm{MHz}$. The investigation revealed that reflection loss from snow is more dependent from the frequency than from the thickness of snow coverage. Amongst mobile communication bands, the strongest microwave reflection (reflection loss only 3.2dB) was registered at WiFi2G band. Averaging all the snow thicknesses, the highest reflection loss occurs at GSM1800UL (1710-1785 MHz) and UMTS2100DL (2110-2170 MHz) bands. The least attenuation of reflection occurs in adjacent DECT band.
\end{abstract}

Keywords: electromagnetic fields, radiofrequency, microwaves, reflection, reflectance, snow, water, mobile phones.

\section{INTRODUCTION}

Having a proper risk management of electromagnetic fields (EMFs) requires accounting also seasonal factors which may affect the propagation of electromagnetic fields. The microwave reflectance or transmittance of water depends greatly on the frequency, but also on the state of the water. Water allows much less microwave penetration depth as compared to the ice. Water in semi-solid state, such as snow is an important issue of risk management especially in Nordic countries where in winter months snow precipitation and coverage may significantly affect the propagation of microwaves. The important issue is to assess the radiofrequency $(\mathrm{RF})$ radiation levels from the point of human safety: in work environment - the occupational safety and in open places, schools etc. the public safety. The propagation of radiowaves may be of issue in case of seasonal factors near radiofrequency sources, such as antennas of TV and radio broadcasting, emergency services networks, and mobile communications. Although TV and radio antennas are not so common in the technogenic environment, developments in mobile communications has placed thousands of microwave antennas in the immediate vicinity where people work, live and conduct their everyday activities. Many of these antennas are not tower placed but positioned on the buildings, on the floors and on the rooftops (an example is presented in figures 1 and 2). Where safety of people needs to be considered, it is important to assess the effect of snow to radiowave propagation at close proximity to the radiofrequency source. Assessing RF safety is also relevant with respect to communication signal transmittance, as seasonal factors may attenuate the signal, hindering the communication links operability. Such cases may include TV and radio broadcasting antennas, but even in more cases private and business mobile communications such as walkie-talkies, DECT phones, wireless data, voice and video links. In most cases public communications operability may be substituted with other means of communications, whereas in emergency services and military operations the reliability of means of communication plays a crucial role, hence characterizing the critical effect of seasonal factors on radiowave propagation.

In 2016 new occupational electromagnetic fields' legislation has emerged in European Union member states, consequent to the European Parliament and the Council of the European Union issuing a new directive 2013/35/EU [1], [2]. The new legislation sets new standards for the electromagnetic fields' risk assessment and risk management. Amongst other duties, the obligation of the employer is to reduce the risks from electromagnetic fields to a minimum. This 
entails implementing technical and administrative means to reduce the workers exposure to the electromagnetic fields and to arrange the work in a way where minimal amount of exposure is attained.

In assessing the safety of workers and other people nearby the antennas, the risk assessment is likely to be carried out under conditions without snow interaction with the surrounding surfaces. In unforeseen cases, where snow would affect the reflection properties of the walls, floors and ground plane surrounding the high power transmission antenna, the microwave exposure levels may be significantly elevated. Workers working under the conditions of elevated electromagnetic fields levels are subjected to an increased health and/or safety risk. Therefore, the risk assessment should be always carried out under worst case scenario conditions, including the reflective properties of the surfaces surrounding the antenna.

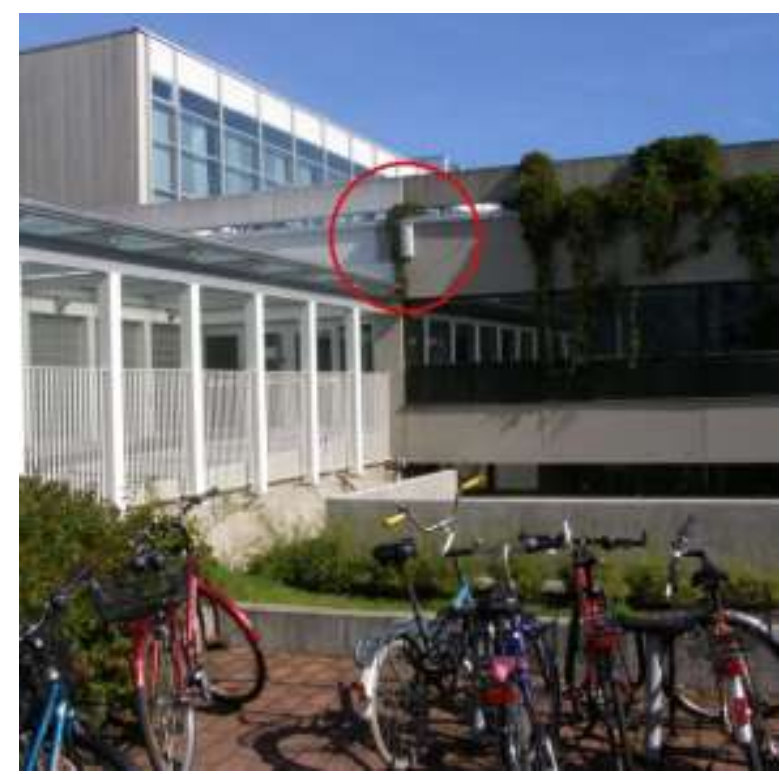

Fig. 1. An example of a mobile communications base station antenna (marked with a red circle), positioned close to ground and building surfaces on top of the entrance; Tampere University campus, Finland.

As the excess water in and on the building material so could snow alter the interaction of the microwave with the material. The reflective or absorptive properties of the surfaces could be emphasized, resulting in a new electromagnetic wave propagation scenario in the vicinity of the material's surface. Such cases may be present at locations of high power mobile communications and public broadcasting antennas.

In assessing microwave propagation and interaction with materials, basically three types of behavior can be observed: transmission, reflection and absorption. Materials transparent to microwaves have low dielectric loss, hence microwaves pass through it with little or no attenuation. Microwaves reflect back from microwave opaque materials and no transmission occurs. In absorbing materials, high dielectric loss causes microwave absorption within the material, where the level of absorption is dependent on the dielectric loss factor. Absorption is directly related to transmission and reflection, where both are reduced and the microwave energy is absorbed within the material and turned into heat [3][5].

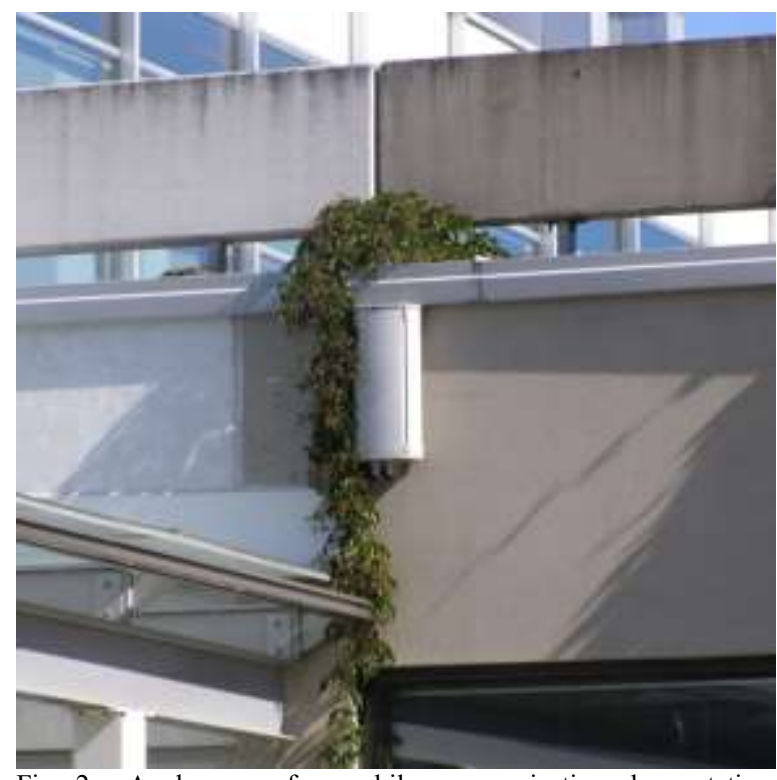

Fig. 2. A close-up of a mobile communications base station antenna, positioned close to ground and building surfaces; Tampere University campus, Finland.

Electrically conductive surfaces cause the reflection, where the microwave is reflected from the surface and/or from the inside layers of the material. The capacitive and conductive components of the dielectric response of the material is determined by the dielectric constant and the dielectric loss factor [4].

\section{METHOD}

In this study fresh snow microwave reflectance properties were investigated. The measurement environmental conditions were: relative humidity 82 $87 \%$, air temp. $-0.9 \ldots-1.1^{\circ} \mathrm{C}$; snow temp. $-1.5 \ldots-$ $1.7^{\circ} \mathrm{C}$. The fresh snow (figure 4) was in small fine particles as the testing was performed within 8 hours after snowfall.

In order to conduct the snow measurements, outside temporary laboratory was assembled. The measurement setup included Rohde\&Schwarz Universal Protocol Tester CRTU-RU capable of generating and measuring signals up to $2700 \mathrm{MHz}$, with a maximum output power of $13 \mathrm{dBm}$. Two standard gain horn antennas were used as presented in figure 3. The Tx antenna transmitted the different radiowave wavelengths, whereas $\mathrm{Rx} 1$ received the reflected wave. 
From the transmitting horn antenna, the microwave propagates from the horn antenna in a parallel beam, which is called the near-field or also Fresnel zone. Field divergence happens in the socalled far-field or Fraunhöfer zone and the wave intensity decreases by the inverse square law - the amplitude decreases in inverse proportion to distance from the aperture of the horn [6].

The distance of the near field zone (1) in case of rectangular horn opening is determined by the formula by Botsco [7] (formula 1): $A$ is the dimension of the largest side of the rectangle and $\lambda$ the wavelength. The antennas were distanced in a manner to place the sample to the far field region where the microwave hit the material.

$$
l=\frac{A^{2}}{2 \lambda}
$$

The snow was placed on a sample holder $60 \times 60$ $\mathrm{cm}$ in dimension and $40 \mathrm{~cm}$ in height, casket made of RF-invisible material (figures 5, 6). The transmitting antenna was placed underneath the tray, as so the reflection receiving antenna $\mathrm{Rx} 1$. The setup was surrounded by RF absorbing elements in order to reduce the inbound and outbound radiowaves. The snow was measured at different thicknesses: 2, 5, 10, $15,20,25,30,35,40$ centimeters.

The snow was not damped, accumulating thickness of snow layers was placed in a light way, approximating the natural fall and packing of snow on the ground.

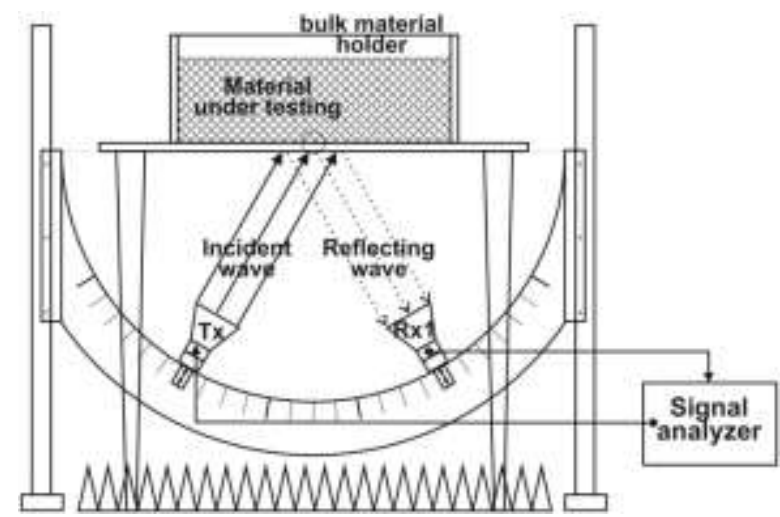

Fig. 3. Measurement setup included irradiator horn antenna Tx and two receiving horn antennas: $\mathrm{Rx} 1$ for reflected wave and $\mathrm{Rx} 2$ for transmitted wave measurement. 
snow sample as a logarithmic ratio of powers in decibels $(\mathrm{dB})$, which is a ratio of the reflected $\left(\mathrm{P}_{\mathrm{r}}\right)$ wave to the full reflection $\left(\mathrm{P}_{\mathrm{i}}\right)$. The measurements were registered in unit of measure powers decibel milliwatts $(\mathrm{dBm})$. Ratio in decibels $(\mathrm{dB})$ is a representation of gain or loss of the material under testing (MUT). Subtracting two powers $(\mathrm{dBm})$ is resulting in their ratio $(\mathrm{dB})$; reflection loss $(\mathrm{RL})$ as presented in formula 2 .

$$
R L(\mathrm{~dB})=10 \cdot \log _{10}\left(\frac{P_{r}}{P_{i}}\right) d B=P_{r}\left|d B m-P_{i}\right| d B m
$$

The measurements covered radiofrequencies from $1700-2700 \mathrm{MHz}$ which accounts most of the mobile communication bands including, GSM1800, DECT, UMTS2100, WIFI2G, LTE2600. It should be noted that in some countries the mobile operators are allowed to use whatever frequency bands they have acquired to whatever protocol they wish to transmit at these frequencies. Therefore, in example the $4 \mathrm{G} \mathrm{LTE}$ protocol could be encountered in any of the frequency bands. The output power was $10 \mathrm{dBm}(10 \mathrm{~mW})$.

The analysis was conducted for each single frequency point within the span range, accounting reflection loss in decibels $(\mathrm{dB})$ as presented in formula 2 . The $0 \mathrm{~dB}$ reflection loss would mean total reflection of the radiowave from the sample, whereas $10 \mathrm{~dB}$ would characterize the loss of power of tenfold due to the material.

The analysis presents mobile communication band specific average reflection losses for the following bands: GSM1800UL (1710-1785 MHz), GSM1800DL (1805-1880 MHz), DECT (1880-1900 $\mathrm{MHz})$ UMTS2100UL (1920-1980 MHz), UMTS2100DL (2110-2170 MHz), WiFi2G (2400$2484 \mathrm{MHz})$, LTE2600UL (2500-2570 MHz), LTE2600DL (2620-2690 MHz); the analysis treats mobile communications upload (UL) and download (DL) bands separately, as download links i.e. mobile phone base station antenna frequencies, are of most interest, due to the relatively higher output power as compared to upload units (e.g. mobile phones). LTE800 and GSM900 mobile bands were omitted from the investigation due to the frequency limitations of the current setup.

\section{RESULTS}

Snow microwave reflection properties were determined on nine different snow thicknesses. Results averaged across the sample, show a noticeable variation in the reflected microwave levels as a result of snow thickness and the frequency.

The extremities of the reflection loss were determined by 1) removing the sample and 2) replacing the sample with a fully reflective Aluminum sheet. The lowest reflection loss was registered 2.6 $\mathrm{dB}$ and the highest reflection loss 29.9 $\mathrm{dB}$ (figure 7).
The investigation revealed that reflection loss from snow is more dependent from the frequency than from the thickness of snow coverage, whereas the highest variation in reflection loss was registered at WiFi2G band (2400-2484 MHz). The main reflection attenuation effect lies within the structure and dielectric properties of the snow particles, and not so much in the bulk condition of snow.

Figure 9 presents mobile communications band specific reflection loss distributed into fresh snow thicknesses' subgroups. The highest reflection loss occurs at GSM1800UL and UMTS2100DL bands. The least attenuation of reflection occurs in adjacent DECT band. The variation between the lowest reflection and highest reflection of power across the frequency span of 1700-2700 MHz was about $27 \mathrm{~dB}$ across all snow thicknesses.

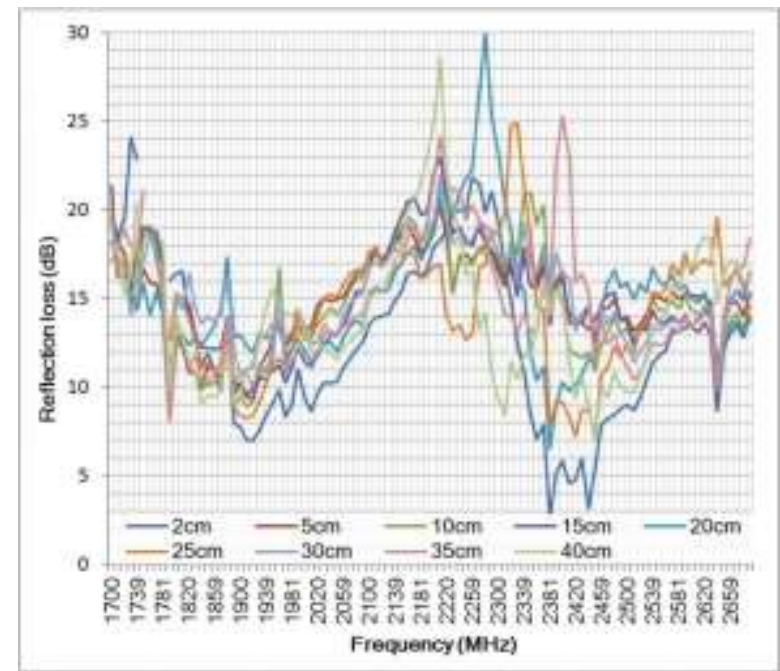

Fig. 7. Reflection loss as the function of frequency, distributed across different fresh snow thicknesses.

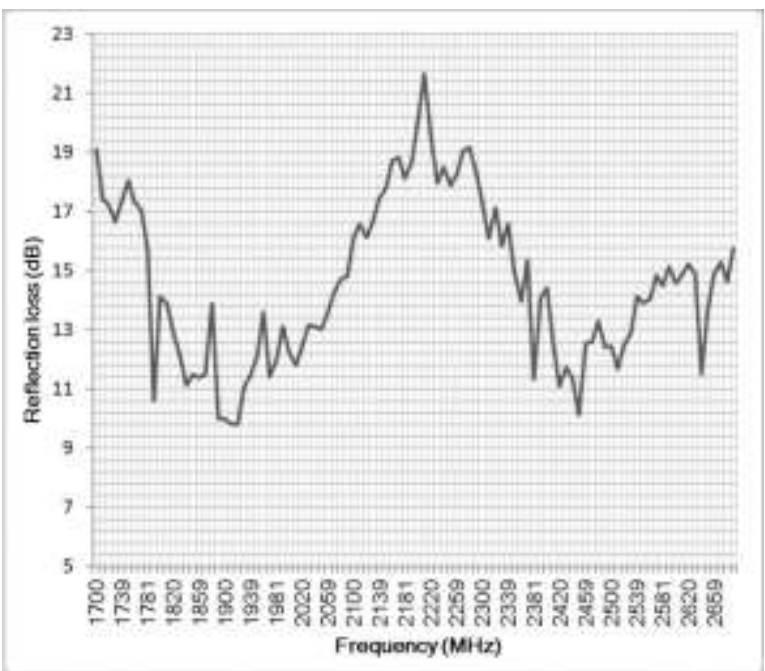

Fig. 8. Averaged reflection loss across all fresh snow thicknesses as a function of frequency. 


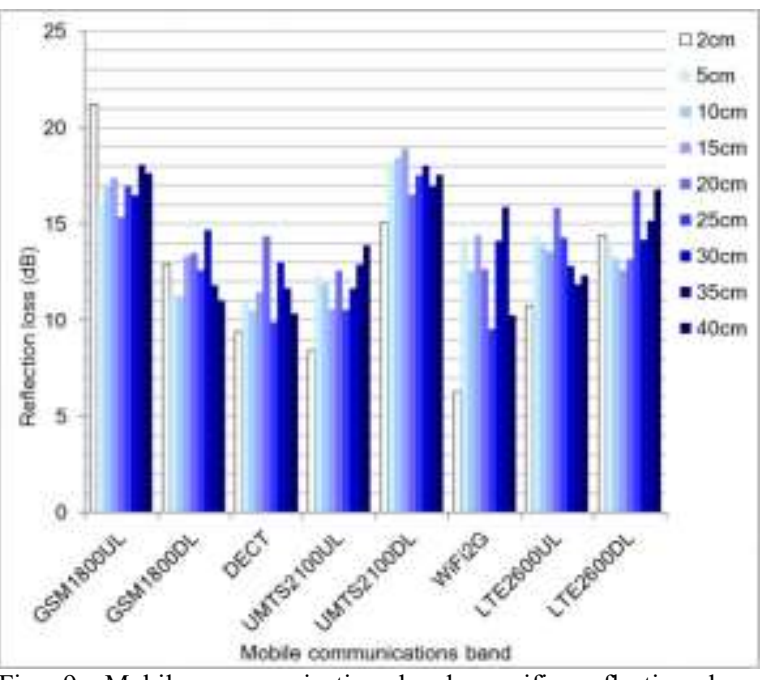

Fig. 9. Mobile communication band specific reflection loss distributed into subgroups of snow thickness of 2 to $40 \mathrm{~cm}$.

Table 1 presents mean and lowest reflection loss per mobile communications band as averaged across different thicknesses of fresh snow $(2$ to $40 \mathrm{~cm}$ ). A strong microwave reflection (reflection loss only $3.2 \mathrm{~dB}$ ) was registered at WiFi2G band; also a moderate microwave reflection occurs at DECT and UMTS2100UL bands.

Figure 8 presents a combined reflection loss curve where reflection loss of all snow thicknesses was averaged. At 2.1 to $2.3 \mathrm{GHz}$ frequency span the fresh snow reflection attenuation is most effective.

Table I

Mobile communications band specific reflection loss $(\mathrm{dB})$ of snow

\begin{tabular}{|l|c|c|c|}
\hline \multirow{2}{*}{$\begin{array}{c}\text { Mobile } \\
\text { communications } \\
\text { band }\end{array}$} & $\begin{array}{c}\text { Frequency span } \\
(\mathrm{MHz})\end{array}$ & \multicolumn{2}{|c|}{ Snow reflection loss $(\mathrm{dB})$} \\
\cline { 3 - 4 } & & mean & lowest \\
\hline GSM1800UL & $1710-1785$ & 17.1 & 14.1 \\
\hline GSM1800DL & $1805-1880$ & 12.5 & 9.0 \\
\hline DECT & $1880-1900$ & 11.3 & 7.7 \\
\hline UMTS2100UL & $1920-1980$ & 11.6 & 7.0 \\
\hline UMTS2100DL & $2110-2170$ & 17.5 & 14.0 \\
\hline WiFi2G & $2400-2484$ & 12.2 & 3.2 \\
\hline LTE2600UL & $2500-2570$ & 13.3 & 8.7 \\
\hline LTE2600DL & $2620-2690$ & 12.1 & 8.7 \\
\hline
\end{tabular}

\section{CONCLUSIONS AND DISCUSSION}

Although the total reflection (reflection loss of 0 $\mathrm{dB}$ ) was not registered during the snow microwave measurements, a large variation in between the lowest and highest reflection loss was determined across the sample. This is a significant indicator of snow alteration of microwave propagation: both the attenuation of the signal and elevation of exposure could occur under different snow coverage conditions.
The results demonstrate that next to building materials, also snow coverage is a considerable factor in assessing microwave propagation near the RF sources. The safety of the workers in working environment and people at public places should be assessed in the electromagnetic fields' risk assessment considering also the seasonal conditions, including the snow coverage of different thicknesses. The current study has demonstrated that snow coverage may significantly affect the microwave exposure level at open spaces where snow precipitation could result in snow coverage in the proximity of RF sources. Assessing the effect of snow coverage on microwave propagation is critical where RF sources, such as mobile phone base station antennas are placed on buildings or other structures made of RF-transparent materials. In such locations the safety of people may have been assessed based on the fact that radiowaves penetrate building materials, hence reflections are minimized. In case of snow precipitation, the resulting amplification of microwaves may create standing waves, where summation of the waves occurs, hence multiplying nearby exposure levels.

The extent of the reflection loss is due to the absorption effect of microwave into water molecules. Future studies should focus on different states of snow, as dependent on the age and weather treatment, the structure of snow could vary significantly, also altering the properties of interaction of snow and the microwave.

\section{REFERENCES}

[1] TheEuropean Parliament and the Council, Directive 2013/35/EU on the minimum health and safety requirements regarding the exposure of workers to the risks arising from physical agents (electromagnetic fields), vol. Official J, no. June. 2013, p. L 179/1-21.

[2] Vabariigi Valitsuse määrus 01.04.2016 nr 44, "Töötervishoiu ja tööohutuse nõuded elektromagnetväljadest mõjutatud töökeskkonnale , elektromagnetväljadega kokkupuute piirnormid ja rakendusväärtused ning elektromagnetväljade mõõtmise kord," Riigi Teat., vol. RT I, 07.0, no. 4, 2016.

[3] S. Das, A. K. Mukhopadhyay, S. Datta, and D. Basu, "Prospects of microwave processing: an overview," Bull. Mater. Sci., vol. 32, no. 1, pp. 1-13, 2009.

[4] E. T. Thostenson and T.-W. Chou, "Microwave processing: fundamentals and applications," Compos. Part A Appl. Sci. Manuf., vol. 30, no. 9, pp. 1055-1071, 1999.

[5] D. E. Clark, D. C. Folz, and J. K. West, "Processing materials with microwave energy," Mater. Sci. Eng. A, vol. 287, no. 2, pp. 153-158, 2000.

[6] J. Blitz, Electrical and magnetic methods of non-destructive testing, vol. 3. Springer Science \& Business Media, 2012.

[7] R. J. Botsco, R. W. Cribbs, R. J. King, and R. C. McMaster, "Microwave methods and applications in nondestructive testing," Nondestruct. Test. Handb., vol. 4, 1986. 